\title{
Distributed Continuous-time Optimization with Scalable Adaptive Event-based Mechanisms
}

DOI:

10.1109/TSMC.2018.2867175

\section{Document Version}

Accepted author manuscript

Link to publication record in Manchester Research Explorer

\section{Citation for published version (APA):}

Wu, Z., Li, Z., Ding, Z., \& Li, Z. (2018). Distributed Continuous-time Optimization with Scalable Adaptive Eventbased Mechanisms. IEEE Transactions on Systems, Man and Cybernetics: Systems .

https://doi.org/10.1109/TSMC.2018.2867175

\section{Published in:}

IEEE Transactions on Systems, Man and Cybernetics: Systems

\section{Citing this paper}

Please note that where the full-text provided on Manchester Research Explorer is the Author Accepted Manuscript or Proof version this may differ from the final Published version. If citing, it is advised that you check and use the publisher's definitive version.

\section{General rights}

Copyright and moral rights for the publications made accessible in the Research Explorer are retained by the authors and/or other copyright owners and it is a condition of accessing publications that users recognise and abide by the legal requirements associated with these rights.

\section{Takedown policy}

If you believe that this document breaches copyright please refer to the University of Manchester's Takedown Procedures [http://man.ac.uk/04Y6Bo] or contact uml.scholarlycommunications@manchester.ac.uk providing relevant details, so we can investigate your claim.

\section{OPEN ACCESS}




\title{
Distributed Continuous-time Optimization with Scalable Adaptive Event-based Mechanisms
}

\author{
Zizhen Wu, Zhenhong Li, Zhengtao Ding, and Zhongkui Li
}

\begin{abstract}
This paper investigates the distributed continuoustime optimization problem, which consists of a group of agents with variant local cost functions. An adaptive consensus-based algorithm with event triggering communications is introduced, which can drive the participating agents to minimize the global cost function and exclude the Zeno behavior. Compared to the existing results, the proposed event-based algorithm is independent of the parameters of the cost functions, using only the relative information of neighboring agents, and hence is fully distributed. Furthermore, the constraints of the convexity of the cost functions are relaxed.
\end{abstract}

Index Terms-Distributed optimization, event-triggered control, cooperative control, adaptive control.

\section{INTRODUCTION}

Distributed optimization problem (DOP) has been a vibrant research field over the past decade for its wide applications in a variety of scenarios, including statistical machine learning, economic dispatch of power system over networks, distributed parameter estimation [1], [2], [3], [4], [5]. The study of DOP paves the way for the development of other cooperative control problems discussed as in, e.g., [6], [7], [8], [9], [10], [11], [12], [13]. This paper considers a representative class of DOP, where the agents in the network collaborate to minimize the global objective function, which is the sum of local cost functions. Each of local cost functions is available to the corresponding agent only.

While many existing algorithms are discrete-time, see, e.g., [5], [14] and related references, the continuous-time DOP algorithms have also attracted significant attention recently; see [15], [16], [17], [18]. In particular, the consensus-based gradient methods are studied by many scholars from different perspectives. For instance, the theories provided in [15] can solve the convex optimization problems cooperatively, where the agents are abstracted as simple dynamics model and equip with local gradient sensing abilities. In [16], the authors propose a continuous-time zero-gradient-sum algorithm to acquire the optimal solution with consensus-based strategy. By increasing the communication and computation overhead for the introducing of extra auxiliary variables, the methods in [17] improve the convergence character. The DOP for multiagent system with high-order linear model is analyzed in [18]. The adaptive algorithms in [19] successfully solve DOP with

Z.Z. Wu and Z.K. Li are with Department of Mechanics and Engineering Science, College of Engineering, Peking University, Beijing 100871, China. E-mail: wuzizhen@pku.edu.cn; zhongkli@pku.edu.cn

Z.H. Li and Z.T. Ding are with School of Electrical and Electronic Engineering, University of Manchester, Manchester M13 9PL, UK. E-mail: zhenhong.lidpostgrad.manchester.ac.uk; zhengtao.ding@manchester.ac.uk non convex local cost functions without the knowledge of connectivity on directed graphs.

In the aforementioned papers on continuous-time DOP, the information exchanges among agents require continuous communication processes. Since the bandwidth and power of the network system are limited source in most circumstances, the event-based control strategy is raised as an effective remedy [20], [21]. Continuous-time algorithms for DOP with event-based communication mechanisms is characterized by [22], [23]. Nevertheless, as the authors mentioned in [22], [23], the algorithm parameters to be designed need to satisfy some conditions that contain global information of the network topology, which may turn out to be undesirable for some applications. Therefore, how to remove the limitation of requiring global information in the designed distributed eventbased optimization algorithm is still an open problem.

This paper intends to solve the continuous-time DOP by proposing a fully distributed consensus-based algorithm with event-triggered communication mechanisms. Since only sampled local information between neighboring agents is available, the adaptive algorithm with continuous communications in [19] is not applicable. The design of adaptive DOP algorithms with event-triggered mechanisms faces new challenges, such as the nonlinearity of local gradient, the coupling among the real-time states, sampled states at the communication moment, and the internal states. By dynamically updating the parameters using sampled local information determined by the triggering function, we present a distributed adaptive event-based algorithm, which can drive the participating agents to minimize the global cost function and exclude the Zeno behavior, i.e., an infinite number of triggered events in a finite period of time. Compared to the existing related works, one main contribution of the proposed adaptive event-triggered algorithm is that it is fully distributed, which is independent of either the knowledge of local cost functions or any global information about the network topology. Furthermore, we can establish the convergence analysis for the general case where the local cost functions can be nonconvex, relaxing the constraint of the convexity of the local cost functions in, e.g., [22], [23].

The remainder of this paper is organized as follows. The continuous-time event-based DOP is formulated in Section II. The main results are presented in Section III. In Section IV, illustration are conducted by numerical simulation. Conclusions are drawn in Section V. 


\section{PROBLEM Formulation}

The system model is considered as a group of $N$ agents. For all the agents in this network, assign each of them a local cost function $f_{i}(\mathbf{s}): \mathbf{R}^{n} \rightarrow \mathbf{R}$. The sum of them $f(\mathbf{s})=$ $\sum_{i=1}^{N} f_{i}(\mathbf{s})$ is defined as the global cost function. The intention of this paper is proposing a distributed algorithm to ensure the agents cooperatively solve the following optimization problem:

$$
\min _{\mathbf{s} \in \mathbf{R}^{n}} f(\mathbf{s}) \text {. }
$$

For this purpose, we make the following assumption about the cost functions mentioned before.

Assumption 1: For each agent $i$, the local cost function $f_{i}(\mathbf{s})$ is first-order continuously differentiable and its gradient $\nabla f_{i}(\mathbf{s})$ is locally Lipschitz on $\mathbf{R}^{n}$, namely, there always exists $M_{i} \in \mathbf{R}_{>0}$ meet the inequality that $\left|\nabla f_{i}(\mathbf{x})-\nabla f_{i}(\mathbf{y})\right| \leq$ $M_{i}\|\mathbf{x}-\mathbf{y}\|$ for $\mathbf{x}, \mathbf{y} \in \mathcal{S}$, where $\mathcal{S} \subset \mathbf{R}^{n}$ is compact set and $\|\cdot\|$ represents the Euclidean norm. The global cost function $f(\mathbf{s})$ is strictly convex over $\mathbf{R}^{n}$ and first-order continuously differentiable.

In order to solve the DOP (1), the agents need to exchange information over a communication network. The topology of the network is characterized by an undirected graph $\mathcal{G}=$ $(\mathcal{V}, \mathcal{E})$, where $\mathcal{V}=1, \ldots, N$, is the node set and $\mathcal{E} \subseteq \mathcal{V} \times \mathcal{V}$ is the edge set. The elements of $\mathcal{E}$ characterize the interactive relations between the nodes in $\mathcal{V}$. Specifically, an edge $(i, j)$ means that the nodes $i$ and $j$ can exchange information with each other. An undirected graph $\mathcal{G}$ is connected if there exists a path between each pair of distinct nodes. The adjacency matrix $\mathcal{A}$ is defined as $a_{i j}>0$ if $(i, j) \in \mathcal{E}$ and $a_{i j}=0$ otherwise. Corresponding, the Laplacian matrix of $\mathcal{G}$ is defined as $\mathcal{L}_{i i}=\sum_{j \neq i} a_{i j}$ and $\mathcal{L}_{i j}=-a_{i j}, i \neq j$.

Regarding the communication graph, we have the following assumption.

Assumption 2: The topology graph $\mathcal{G}$ is undirected and connected.

Therefore, the Laplacian matrix $\mathcal{L}$ of undirected graph has the following property.

Lemma 1 ([6]): The Laplacian matrix $\mathcal{L}$ has a simple zero eigenvalue and all the other eigenvalues are positive. The corresponding eigenvector of simple eigenvalue is $N$ dimension column vector $\mathbf{1}_{N}$ with all entries equal to one.

To guarantee the unique solution of the DOP (1), the global cost function must be strictly convex throughout this paper. Similarly as did in [15], [17], we can reformulate the problem (1) as

$$
\min _{\mathbf{x}_{i} \in \mathbf{R}^{n}} f(\mathbf{x})=\sum_{i=1}^{N} f_{i}\left(\mathbf{x}_{i}\right), \text { subject to }\left(\mathcal{L} \otimes I_{n}\right) \mathbf{x}=\mathbf{0},
$$

under Assumptions 1 and 2, where $\mathbf{x}_{i}$ is the real-time state variable of the $i$-th agent, the state vector of the whole network is $\mathbf{x}=\left[\mathbf{x}_{1}^{T}, \mathbf{x}_{2}^{T}, \ldots, \mathbf{x}_{N}^{T}\right]^{T}, I_{n}$ represents the $n$ dimension identity matrix, and $\otimes$ denotes the Kronecker product.

The objective is to solve the DOP (1) by designing a distributed consensus-based algorithm with event triggering communications, and excluding the Zeno behavior. In this case, the agents broadcast their information to the neighbors only at aperiodic event time instants and thereby the distributed optimization algorithm is more challenging.

\section{MAIN RESULTS}

Based on sampled information from neighboring agents, a distributed event-triggered coordination control algorithm is designed as

$$
\begin{aligned}
& \dot{\mathbf{x}}_{i}=-\nabla f_{i}\left(\mathbf{x}_{i}\right)-\sum_{j=1}^{N} c_{i j} a_{i j}\left(\tilde{\mathbf{x}}_{i}-\tilde{\mathbf{x}}_{j}\right)-\mathbf{v}_{i} \\
& \dot{\mathbf{v}}_{i}=\sum_{j=1}^{N} c_{i j} a_{i j}\left(\tilde{\mathbf{x}}_{i}-\tilde{\mathbf{x}}_{j}\right), \\
& \dot{c}_{i j}=k_{i j} a_{i j}\left(\tilde{\mathbf{x}}_{i}-\tilde{\mathbf{x}}_{j}\right)^{T}\left(\tilde{\mathbf{x}}_{i}-\tilde{\mathbf{x}}_{j}\right)
\end{aligned}
$$

where $\tilde{\mathbf{x}}_{i}(t)=\mathbf{x}_{i}\left(t_{k}^{i}\right), \forall t \in\left[t_{k}^{i}, t_{k+1}^{i}\right)$, denotes the communication moment state and $\mathbf{v}_{i}$ is the auxiliary variable of the $i-t h$ agent, the time-varying coupling coefficient $c_{i j}$ for the edge $(i, j)$ satisfies $c_{i j}(0)=c_{j i}(0)$, and $k_{i j}=k_{j i}$ are positive constants.

In (3), $t_{k}^{i}$ denotes the $k$-th event triggering instant of the $i-$ th agent, which is determined by the following event triggering function:

$$
\begin{aligned}
T_{i}(t)= & \sum_{j=1}^{N}\left(1+\sigma c_{i j}\right) a_{i j} \mathbf{e}_{i}^{T} \mathbf{e}_{i}-\mu e^{-\nu t} \\
& -\frac{1}{4} \sum_{j=1}^{N} a_{i j}\left(\tilde{\mathbf{x}}_{i}-\tilde{\mathbf{x}}_{j}\right)^{T}\left(\tilde{\mathbf{x}}_{i}-\tilde{\mathbf{x}}_{j}\right),
\end{aligned}
$$

where $\mathbf{e}_{i}(t)$ represents the measurement error of the $i-t h$ agent, defined as $\mathbf{e}_{i}(t) \triangleq \tilde{\mathbf{x}}_{i}(t)-\mathbf{x}_{i}(t), i=1, \ldots, N$, and $\mu, \nu$ and $\sigma$ are all prescribed positive constants. The event sequence $\left\{t_{k}^{i}\right\}$ represents the time instants that node $i$ updates its controller and sends its present information to its neighbors. The sequence $\left\{t_{k}^{i}\right\}$ is defined as $t_{k+1}^{i}=\inf \left\{t>t_{k}^{i} \mid T_{i}(t) \geq\right.$ $0\}$. At each event instant, the measurement error $\mathbf{e}_{i}(t)$ will be reset to zero.

The compact form can be written as

$$
\begin{aligned}
& \dot{\mathbf{x}}=-\nabla f(\mathbf{x})-\left(\mathcal{L}_{c} \otimes I_{n}\right) \tilde{\mathbf{x}}-\mathbf{v}, \\
& \dot{\mathbf{v}}=\left(\mathcal{L}_{c} \otimes I_{n}\right) \tilde{\mathbf{x}}, \\
& \dot{c}_{i j}=k_{i j} a_{i j}\left(\tilde{\mathbf{x}}_{i}-\tilde{\mathbf{x}}_{j}\right)^{T}\left(\tilde{\mathbf{x}}_{i}-\tilde{\mathbf{x}}_{j}\right),
\end{aligned}
$$

where $\tilde{\mathbf{x}}=\left[\tilde{\mathbf{x}}_{1}^{T}, \cdots, \tilde{\mathbf{x}}_{N}^{T}\right]^{T}, \mathbf{v}=\left[\mathbf{v}_{1}^{T}, \cdots, \mathbf{v}_{N}^{T}\right]^{T}, \nabla f(\mathbf{x})=$ $\left[\nabla f_{1}\left(\mathbf{x}_{1}\right)^{T}, \ldots, \nabla f_{N}\left(\mathbf{x}_{N}\right)^{T}\right]^{T}$, and $\mathcal{L}_{c}$ is defined as $\mathcal{L}_{c}^{i j}=$ $-c_{i j} a_{i j}, i \neq j$, and $\mathcal{L}_{c}^{i i}=\sum_{j=1, j \neq i}^{N} c_{i j} a_{i j}$. Obviously, the matrix $\mathcal{L}_{c}$ with a time-varying weight $c_{i j} a_{i j}$ for each edge $(i, j)$ is also a symmetric Laplacian matrix when the graph $\mathcal{G}$ is undirected.

As the first step in the analysis, we certify the following lemma.

Lemma 2: Under Assumptions 1 and 2, the optimal solution of the DOP (1) is the equilibrium-point of the system (5) when $\sum_{i=1}^{N} \mathbf{v}_{i}(0)=\mathbf{0}$.

Proof 1: Since the network topology is undirected and connected, according to the definition of $\mathcal{L}_{c}$, we can verify the property that $\mathbf{1}_{N}^{T} \mathcal{L}_{c}=\mathbf{0}_{N}$. Therefore,

$$
\left(\mathbf{1}_{N} \otimes I_{n}\right)^{T} \dot{\mathbf{v}}=\left(\mathbf{1}_{N} \otimes I_{n}\right)^{T}\left(\mathcal{L}_{c} \otimes I_{n}\right) \tilde{\mathbf{x}} \equiv \mathbf{0}_{n} .
$$

Then we have

$$
\left(\mathbf{1}_{N} \otimes I_{n}\right)^{T} \mathbf{v}(t)=\left(\mathbf{1}_{N} \otimes I_{n}\right)^{T} \mathbf{v}(0)=\boldsymbol{\vartheta},
$$


where $\boldsymbol{\vartheta} \in \mathbf{R}^{n}$ denotes a constant vector. When $\boldsymbol{\vartheta}=\mathbf{0}$, then the equilibrium point $(\overline{\mathbf{x}}, \overline{\mathbf{v}})$ of (5) satisfies

$$
\begin{gathered}
0=-\nabla f(\overline{\mathbf{x}})-\left(\mathcal{L}_{c} \otimes I_{n}\right) \overline{\mathbf{x}}-\overline{\mathbf{v}}, \\
0=\left(\mathcal{L}_{c} \otimes I_{n}\right) \overline{\mathbf{x}} .
\end{gathered}
$$

Through Lemma 2, we can obtain that the vector $\overline{\mathbf{x}}$ belongs to the zero space of $\mathcal{L}_{c}$ from (9). Thus, $\overline{\mathbf{x}}=\mathbf{1}_{N} \otimes \varsigma$, where $\varsigma \in \mathbf{R}^{n}$ is a constant vector. Multiplying (8) by $\left(\mathbf{1}_{N} \otimes I_{n}\right)^{T}$ from the left side and using (7), we can obtain that $\Sigma_{i=1}^{N} \nabla f_{i}\left(\overline{\mathbf{x}}_{i}\right)=0$. Then the optimality condition of the system (5), i.e., $\nabla f\left(\mathbf{x}^{*}\right)=0$ is satisfied, which, by noting the strict convexity of $f$, means that $\overline{\mathbf{x}}=\mathbf{x}^{*}$ is uniquely determined. Consequently, we have $\overline{\mathbf{v}}_{i}=-\nabla f_{i}\left(\mathbf{x}_{\mathbf{i}}{ }^{*}\right)$. This completes the proof.

Based on the aforementioned analysis, we are well ready to present the main results of this paper.

Theorem 1: Provided that Assumptions 1 and 2 hold, the algorithm (3) drives the state of involved agents asymptotically converge to the optimazation of the DOP (2) and achieve consensus with the initial condition $\sum_{i=1}^{N} \mathbf{v}_{i}(0)=\mathbf{0}$.

Proof 2: Firstly, we apply a state transformation to transfer the equilibrium point $(\overline{\mathbf{x}}, \overline{\mathbf{v}})$ of (5) to zero. Let $\varphi=\mathbf{x}-\overline{\mathbf{x}}$, $\psi=\mathbf{v}-\overline{\mathbf{v}}$. Analogously, we have $\tilde{\varphi}=\varphi+\mathbf{e}$, hence, $\tilde{\varphi}=$ $\tilde{\mathbf{x}}-\overline{\mathbf{x}}$. Then, we can rewrite (5) as

$$
\begin{aligned}
& \dot{\boldsymbol{\varphi}}=-\mathbf{g}-\left(\mathcal{L}_{c} \otimes I_{n}\right) \tilde{\boldsymbol{\varphi}}-\boldsymbol{\psi}, \\
& \dot{\boldsymbol{\psi}}=\left(\mathcal{L}_{c} \otimes I_{n}\right) \tilde{\boldsymbol{\varphi}}, \\
& \dot{c}_{i j}=k_{i j} a_{i j}\left(\tilde{\mathbf{x}}_{i}-\tilde{\mathbf{x}}_{j}\right)^{T}\left(\tilde{\mathbf{x}}_{i}-\tilde{\mathbf{x}}_{j}\right),
\end{aligned}
$$

where $\mathbf{g}=\nabla f(\mathbf{x})-\nabla f(\overline{\mathbf{x}})$. Evidently, the proposed algorithm (3) can solve the DOP (2), if $\lim _{t \rightarrow \infty} \boldsymbol{\varphi}(t)=\mathbf{0}$, meanwhile, $\lim _{t \rightarrow \infty} \boldsymbol{\psi}(t)=\mathbf{0}$.

Next, consider the Lyapunov functions candidate:

$$
\begin{aligned}
V_{1}= & \frac{1}{2} \phi \boldsymbol{\varphi}^{T} \boldsymbol{\varphi}+\frac{1}{2}(\boldsymbol{\varphi}+\boldsymbol{\psi})^{T}(\boldsymbol{\varphi}+\boldsymbol{\psi}) \\
& +\sum_{i=1}^{N} \sum_{j=1, j \neq i}^{N} \frac{\left(\sqrt{\phi} c_{i j}-\frac{1}{\sqrt{\phi}} \alpha\right)^{2}}{8 k_{i j}}
\end{aligned}
$$

where $\phi$ and $\alpha$ are positive scalars to be designed later. Definitely, $V_{1}$ is positive definite.

The time derivative of the Lyapunov function $V_{1}$ along the trajectory of (11) can be written as

$$
\begin{aligned}
\dot{V}_{1}= & (\phi+1) \boldsymbol{\varphi}^{T} \dot{\boldsymbol{\varphi}}+\boldsymbol{\varphi}^{T} \dot{\boldsymbol{\psi}}+\boldsymbol{\psi}^{T} \dot{\boldsymbol{\varphi}}+\boldsymbol{\psi}^{T} \dot{\boldsymbol{\psi}} \\
& +\sum_{i=1}^{N} \sum_{j=1, j \neq i}^{N} \frac{2\left(\phi c_{i j}-\alpha\right)}{8 k_{i j}} \dot{c}_{i j} \\
= & -(\phi+1) \boldsymbol{\varphi}^{T} \mathbf{g}-\phi \boldsymbol{\varphi}^{T}\left(\mathcal{L}_{c} \otimes I_{n}\right) \tilde{\boldsymbol{\varphi}}-(\phi+1) \boldsymbol{\varphi}^{T} \boldsymbol{\psi} \\
& -\boldsymbol{\psi}^{T} \mathbf{g}-\boldsymbol{\psi}^{T} \boldsymbol{\psi}+\sum_{i=1}^{N} \sum_{j=1, j \neq i}^{N} \frac{\left(\phi c_{i j}-\alpha\right)}{4 k_{i j}} \dot{c}_{i j} .
\end{aligned}
$$

By using the facts that $\mathbf{x}_{i}-\mathbf{x}_{j}=\varphi_{i}-\boldsymbol{\varphi}_{j}, \tilde{\mathbf{x}}_{i}-\tilde{\mathbf{x}}_{j}=\tilde{\varphi}_{i}-\tilde{\varphi}_{j}$, and $\mathbf{e}_{i}=\tilde{\mathbf{x}}_{i}-\mathbf{x}_{i}$, we can obtain that

$$
\begin{aligned}
& -\boldsymbol{\varphi}^{T}\left(\mathcal{L}_{c} \otimes I_{n}\right) \tilde{\boldsymbol{\varphi}} \\
= & -\tilde{\mathbf{x}}^{T}\left(\mathcal{L}_{c} \otimes I_{n}\right) \tilde{\mathbf{x}}+\mathbf{e}^{T}\left(\mathcal{L}_{c} \otimes I_{n}\right) \tilde{\mathbf{x}} \\
\leq & -\tilde{\mathbf{x}}^{T}\left(\mathcal{L}_{c} \otimes I_{n}\right) \tilde{\mathbf{x}}+\frac{1}{2} \tilde{\mathbf{x}}^{T}\left(\mathcal{L}_{c} \otimes I_{n}\right) \tilde{\mathbf{x}}+\frac{1}{2} \mathbf{e}^{T}\left(\mathcal{L}_{c} \otimes I_{n}\right) \mathbf{e} \\
= & -\frac{1}{2} \tilde{\varphi}^{T}\left(\mathcal{L}_{c} \otimes I_{n}\right) \tilde{\boldsymbol{\varphi}}+\frac{1}{2} \mathbf{e}^{T}\left(\mathcal{L}_{c} \otimes I_{n}\right) \mathbf{e} .
\end{aligned}
$$

Substituting (13) into (12) yields

$$
\begin{aligned}
& \dot{V}_{1}=-\frac{(\phi+1)}{2} \boldsymbol{\varphi}^{T} \mathbf{g}-\phi \boldsymbol{\varphi}^{T}\left(\mathcal{L}_{c} \otimes I_{n}\right) \tilde{\varphi}+\frac{1}{4}\|\mathbf{g}\|^{2} \\
& +\frac{1}{4}(\phi+1)^{2} \boldsymbol{\varphi}^{T} \boldsymbol{\varphi}-\left\|\frac{\mathbf{g}}{2}+\frac{(\phi+1) \boldsymbol{\varphi}}{2}+\boldsymbol{\psi}\right\|^{2} \\
& +\frac{\phi}{4} \sum_{i=1}^{N} \sum_{j=1}^{N} c_{i j} a_{i j}\left(\tilde{\mathbf{x}}_{i}-\tilde{\mathbf{x}}_{j}\right)^{T}\left(\tilde{\mathbf{x}}_{i}-\tilde{\mathbf{x}}_{j}\right) \\
& -\frac{\alpha}{4} \sum_{i=1}^{N} \sum_{j=1}^{N} a_{i j}\left(\tilde{\mathbf{x}}_{i}-\tilde{\mathbf{x}}_{j}\right)^{T}\left(\tilde{\mathbf{x}}_{i}-\tilde{\mathbf{x}}_{j}\right) \\
& \leq-\frac{(\phi+1)}{2} \boldsymbol{\varphi}^{T} \mathbf{g}-\frac{\phi}{2} \tilde{\boldsymbol{\varphi}}^{T}\left(\mathcal{L}_{c} \otimes I_{n}\right) \tilde{\boldsymbol{\varphi}}+\frac{1}{4}\|\mathbf{g}\|^{2} \\
& +\frac{1}{4}(\phi+1)^{2} \boldsymbol{\varphi}^{T} \boldsymbol{\varphi}+\frac{\phi}{2} \mathbf{e}^{T}\left(\mathcal{L}_{c} \otimes I_{n}\right) \mathbf{e} \\
& -\left\|\frac{\mathbf{g}}{2}+\frac{(\phi+1) \boldsymbol{\varphi}}{2}+\boldsymbol{\psi}\right\|^{2}+\frac{\phi}{2} \tilde{\boldsymbol{\varphi}}^{T}\left(\mathcal{L}_{c} \otimes I_{n}\right) \tilde{\boldsymbol{\varphi}} \\
& -\frac{\alpha}{4} \sum_{i=1}^{N} \sum_{j=1}^{N} a_{i j}\left(\tilde{\mathbf{x}}_{i}-\tilde{\mathbf{x}}_{j}\right)^{T}\left(\tilde{\mathbf{x}}_{i}-\tilde{\mathbf{x}}_{j}\right) .
\end{aligned}
$$

Note that

$$
\begin{aligned}
& \sum_{i=1}^{N} \sum_{j=1}^{N} a_{i j}\left(\tilde{\mathbf{x}}_{i}-\tilde{\mathbf{x}}_{j}\right)^{T}\left(\tilde{\mathbf{x}}_{i}-\tilde{\mathbf{x}}_{j}\right) \\
= & \sum_{i=1}^{N} \sum_{j=1}^{N} a_{i j}\left(\mathbf{x}_{i}-\mathbf{x}_{j}\right)^{T}\left(\mathbf{x}_{i}-\mathbf{x}_{j}\right) \\
& +\sum_{i=1}^{N} \sum_{j=1}^{N} a_{i j}\left(\mathbf{e}_{i}-\mathbf{e}_{j}\right)^{T}\left(\mathbf{e}_{i}-\mathbf{e}_{j}\right) \\
& +2 \sum_{i=1}^{N} \sum_{j=1}^{N} a_{i j}\left(\mathbf{x}_{i}-\mathbf{x}_{j}\right)^{T}\left(\mathbf{e}_{i}-\mathbf{e}_{j}\right) .
\end{aligned}
$$

In light of the well-known Young's inequality [24], we can get

$$
\begin{array}{r}
-\sum_{i=1}^{N} \sum_{j=1}^{N} a_{i j}\left(\mathbf{x}_{i}-\mathbf{x}_{j}\right)^{T}\left(\mathbf{e}_{i}-\mathbf{e}_{j}\right) \\
\leq \frac{1}{4} \sum_{i=1}^{N} \sum_{j=1}^{N} a_{i j}\left(\mathbf{x}_{i}-\mathbf{x}_{j}\right)^{T}\left(\mathbf{x}_{i}-\mathbf{x}_{j}\right) \\
+\sum_{i=1}^{N} \sum_{j=1}^{N} a_{i j}\left(\mathbf{e}_{i}-\mathbf{e}_{j}\right)^{T}\left(\mathbf{e}_{i}-\mathbf{e}_{j}\right),
\end{array}
$$


and

$$
\begin{aligned}
& \sum_{i=1}^{N} \sum_{j=1}^{N} a_{i j}\left(\mathbf{e}_{i}-\mathbf{e}_{j}\right)^{T}\left(\mathbf{e}_{i}-\mathbf{e}_{j}\right) \\
\leq & 2 \sum_{i=1}^{N} \sum_{j=1}^{N} a_{i j} \mathbf{e}_{i}^{T} \mathbf{e}_{i}+2 \sum_{i=1}^{N} \sum_{j=1}^{N} a_{i j} \mathbf{e}_{j}^{T} \mathbf{e}_{j} \\
= & 4 \sum_{i=1}^{N} \sum_{j=1}^{N} a_{i j} \mathbf{e}_{i}^{T} \mathbf{e}_{i} .
\end{aligned}
$$

According to Assumption 1, there exists $M_{i} \in \mathbf{R}_{>0}$ for each gradient such that $\left\|\nabla f_{i}\left(\mathbf{x}_{i}\right)-\nabla f_{i}\left(\overline{\mathbf{x}}_{i}\right)\right\| \leq M_{i}\left\|\mathbf{x}_{i}-\overline{\mathbf{x}}_{i}\right\|, i=$ $1,2, \ldots, N$, for $(\mathbf{x}, \mathbf{v}) \in \mathcal{S}$. Recalling the definition of $\varphi$ and $\mathrm{g}$, we can exploit the fact that $\|\mathbf{g}\|^{2} \leq M_{\max }^{2} \varphi^{T} \boldsymbol{\varphi}$, where $M_{\max }=\max \left(M_{1}, \ldots, M_{N}\right)$. In light of (15), (16), and also the aforementioned facts, we can obtain from (14) that

$$
\begin{aligned}
\dot{V}_{1} \leq & -\frac{1}{2}(\phi+1) \boldsymbol{\varphi}^{T} \mathbf{g}+\frac{\phi}{2} \mathbf{e}^{T}\left(\mathcal{L}_{c} \otimes I_{n}\right) \mathbf{e} \\
& -\left\|\frac{\mathbf{g}}{2}+\frac{(\phi+1) \boldsymbol{\varphi}}{2}+\boldsymbol{\psi}\right\|^{2}-\frac{1}{4} \boldsymbol{\varphi}^{T} \boldsymbol{\varphi} \\
& +\frac{1}{4}\left[(\phi+1)^{2}+M_{m a x}^{2}+1\right] \boldsymbol{\varphi}^{T} \boldsymbol{\varphi} \\
& -\frac{\alpha}{8} \sum_{i=1}^{N} \sum_{j=1}^{N} a_{i j}\left(\tilde{\mathbf{x}}_{i}-\tilde{\mathbf{x}}_{j}\right)^{T}\left(\tilde{\mathbf{x}}_{i}-\tilde{\mathbf{x}}_{j}\right) \\
& -\frac{\alpha}{16} \sum_{i=1}^{N} \sum_{j=1}^{N} a_{i j}\left(\mathbf{x}_{i}-\mathbf{x}_{j}\right)^{T}\left(\mathbf{x}_{i}-\mathbf{x}_{j}\right) \\
& +\frac{\alpha}{8} \sum_{i=1}^{N} \sum_{j=1}^{N} a_{i j}\left(\mathbf{e}_{i}-\mathbf{e}_{j}\right)^{T}\left(\mathbf{e}_{i}-\mathbf{e}_{j}\right) .
\end{aligned}
$$

For further analysis, we define a convex set

$$
\begin{aligned}
\mathcal{C}=\left\{(\mathbf{x}, \mathbf{v}) \in \mathbf{R}^{N n} \times \mathbf{R}^{N n} \mid\right. & \\
& \left.\|\mathbf{x}-\overline{\mathbf{x}}\| \leq\|\mathbf{x}(0)-\overline{\mathbf{x}}\|, \forall \mathbf{v} \in \mathbf{R}^{N n}\right\},
\end{aligned}
$$

which contains the equilibrium point $(\overline{\mathbf{x}}, \overline{\mathbf{v}})$. Since the optimal solution $\mathrm{x}^{*}$ is unique and the set $\mathcal{C}$ is compact for $\mathbf{x}$. For $(\mathbf{x}, \mathbf{v}) \in \mathcal{C}$, we can have $\omega=\varphi(\mathbf{x}(0))^{T} \boldsymbol{\varphi}(\mathbf{x}(0)) \geq$ $\boldsymbol{\varphi}(\mathbf{x}(t))^{T} \boldsymbol{\varphi}(\mathbf{x}(t))$, where $\omega \in \mathbf{R}_{>0}$. When the agents do not achieve consensus yet, there always exists an arbitrary small positive $\delta$ satisfying the inequality: $\sum_{i=1}^{N} \sum_{j=1}^{N} a_{i j}\left(\mathbf{x}_{i}-\right.$ $\left.\mathbf{x}_{j}\right)^{T}\left(\mathbf{x}_{i}-\mathbf{x}_{j}\right) \geq \delta$. The left-hand side item of this inequality equals to zero if and only if the states of all agents are coincident. Hence, a sufficiently large $\alpha$ always can be found to assure that $\frac{\alpha \delta}{4} \geq\left[(\phi+1)^{2}+M_{\max }^{2}+1\right] \cdot \omega$.

By the analysis above, and substituting (17) into inequality (18) yields

$$
\begin{aligned}
\dot{V}_{1} \leq & -\frac{1}{2}(\phi+1) \boldsymbol{\varphi}^{T} \mathbf{g}-\frac{1}{4} \boldsymbol{\varphi}^{T} \boldsymbol{\varphi} \\
& -\left\|\frac{\mathbf{g}}{2}+\frac{(\phi+1) \boldsymbol{\varphi}}{2}+\boldsymbol{\psi}\right\|^{2} \\
& +\frac{\alpha}{2} \sum_{i=1}^{N}\left[\sum_{j=1}^{N}\left(1+\frac{2 \phi}{\alpha \sigma} \cdot \sigma c_{i j}\right) a_{i j} e_{i}^{T} e_{i}\right. \\
& \left.-\frac{1}{4} \sum_{j=1}^{N} a_{i j}\left(\tilde{\mathbf{x}}_{i}-\tilde{\mathbf{x}}_{j}\right)^{T}\left(\tilde{\mathbf{x}}_{i}-\tilde{\mathbf{x}}_{j}\right)\right],
\end{aligned}
$$

where $\phi$ can be any number greater than zero. For the reason that the global objective function $f(\mathbf{x})$ is strictly convex, $\varphi^{T} \mathbf{g}$ will be strictly greater than 0 when $\mathbf{x} \neq \overline{\mathbf{x}}$. And, apparently, $\left\|\frac{\mathrm{g}}{2}+\frac{(\phi+1) \varphi}{2}+\boldsymbol{\psi}\right\|^{2} \geq 0$. By choosing $\alpha \geq \max \left\{\frac{4}{\delta} \cdot[(\phi+\right.$ $\left.\left.1)^{2}+M_{\max }^{2}+1\right] \cdot \omega, \frac{2 \phi}{\sigma}\right\}$ and using the triggering functions (4), it follows form (20) that

$$
\begin{aligned}
\dot{V}_{1} \leq & -\frac{1}{2}(\phi+1) \boldsymbol{\varphi}^{T} \mathbf{g}-\frac{1}{4} \boldsymbol{\varphi}^{T} \boldsymbol{\varphi} \\
& -\left\|\frac{\mathrm{g}}{2}+\frac{(\phi+1) \boldsymbol{\varphi}}{2}+\boldsymbol{\psi}\right\|^{2}+\frac{\alpha}{2} N \mu e^{-\nu t} \\
\leq & -\frac{1}{4} \boldsymbol{\varphi}^{T} \boldsymbol{\varphi}+\frac{\alpha}{2} N \mu e^{-\nu t} .
\end{aligned}
$$

Then, we can obtain from (21) that

$$
0 \leq V_{1}(t) \leq V_{1}(0)+\frac{\alpha}{2} N \mu \int_{0}^{t} e^{-\nu \tau} d \tau .
$$

Therefore, $V_{1}(t)$ is bounded, implying that $\boldsymbol{\varphi}, \boldsymbol{\psi}$ and $c_{i j}$ are all bounded. Since $c_{i j}$ is monotonically increasing, we derive that each $c_{i j}$ converges to some finite values. Recall the fact that $\|\mathbf{g}\|^{2} \leq M_{\max }^{2} \varphi^{T} \boldsymbol{\varphi}$, hence, $\mathbf{g}$ is bounded. Invoking (10), we further know that $\dot{\varphi}$ is bounded too. Besides, (21) enforces that

$$
\begin{aligned}
V_{1}(\infty)-V_{1}(0) \leq & -\frac{1}{4} \int_{0}^{\infty} \varphi^{T}(\tau) \varphi(\tau) d \tau \\
& +\frac{\alpha}{2} N \mu \int_{0}^{\infty} e^{-\nu \tau} d \tau
\end{aligned}
$$

Recast (23), one has

$$
\int_{0}^{\infty} \varphi^{T}(\tau) \varphi(\tau) d \tau \leq 4\left(V_{1}(0)-V_{1}(\infty)+\frac{\alpha}{2 \nu} N \mu\right) .
$$

By Barbalat's lemma [25], using (24) yields, $\varphi$ asymptotically converge to 0 , which further implies that $\mathbf{x} \rightarrow \overline{\mathbf{x}}$ as $t \rightarrow \infty$. In light of the Lemma 2, it is easy to claim that $\mathbf{v} \rightarrow \overline{\mathbf{v}}$ as $t \rightarrow \infty$. This completes the proof.

Remark 1: In the previous related work [22], interesting results are given to solve continuous-time DOP with eventtriggered communications. Nevertheless, it should be noted that the algorithms in [22] are not fully distributed, requiring the knowledge of the local gradients and global connectivity information. By contrast, here, we redesign a modified distributed adaptive event-triggered algorithm, which is independent of either the constant parameters of the cost functions and gradients or any other global information, and therefore is fully distributed. Besides, since the determination of the parameters in (3) and (4) do not depend on the network connectivity, it is not difficult to show that the adaptive algorithm (3) is applicable to the case of dynamically switching topologies.

Remark 2: Different from the previous results in, e.g., [22], [23], in this paper, the constraints on local cost function of convexity and differentiability are further relaxed. As show in Theorem 1, each agent's cost function can be any nonconvex and differentiable functions, and only the global objective function must equip the strict convexity.

The following theorem shows that the Zeno behavior is excluded through the dynamic triggering law.

Theorem 2: Under the event triggering condition (4), the algorithm (3) can preclude the Zeno behavior. 
Proof 3: According to the definition in (4), when $\sum_{j=1}^{N}(1+$ $\left.\sigma c_{i j}\right) a_{i j} \mathbf{e}_{i}^{T} \mathbf{e}_{i}$ exceeds $\frac{1}{4} \sum_{j=1}^{N} a_{i j}\left(\tilde{\mathbf{x}}_{i}-\tilde{\mathbf{x}}_{j}\right)^{T}\left(\tilde{\mathbf{x}}_{i}-\tilde{\mathbf{x}}_{j}\right)+\mu e^{-\nu t}$, then the triggering condition will be satisfied. Consequently, the measurement error $\mathbf{e}_{i}$ is reset to zero and the $i-t h$ agent is triggered to broadcast its information. Hence, each agent will update its control law in the following two cases.

i) During the interval between two adjacent events of agent $i$, the neighbors of the agent are all not be triggered. Combining the definition of $\mathbf{e}_{i}$ with (3), we can write the upper right-hand Dini derivative as

$$
D^{+} \mathbf{e}_{i}(t)=\nabla f_{i}\left(\mathbf{x}_{i}\right)+\sum_{j=1}^{N} c_{i j} a_{i j}\left(\tilde{\mathbf{x}}_{i}-\tilde{\mathbf{x}}_{j}\right)+\mathbf{v}_{i} .
$$

Note that $\mathbf{e}_{i}\left(t_{k}^{i}\right)=0$, hence, the solution of (25) is

$$
\begin{aligned}
\mathbf{e}_{i}(t)= & \int_{t_{k}^{i}}^{t} \nabla f_{i}\left(\mathbf{x}_{i}(\tau)\right) d \tau+\int_{t_{k}^{i}}^{t} \mathbf{v}_{i}(\tau) d \tau \\
& +\int_{t_{k}^{i}}^{t} \sum_{j=1}^{N} c_{i j} a_{i j}\left(\tilde{\mathbf{x}}_{i}(\tau)-\tilde{\mathbf{x}}_{j}(\tau)\right) d \tau .
\end{aligned}
$$

From the proof of Theorem 1, we have that $V_{1}$ is bounded, therefore, we can assume that $\left\|\nabla f_{i}\left(\mathbf{x}_{i}\right)\right\| \leq \bar{\gamma}_{f}$ and $\left\|\mathbf{v}_{i}(t)\right\| \leq$ $\bar{\gamma}_{v}$ hold. Meanwhile we have $c_{i j}$ converge to some positive values, which means that $c_{i j} \leq \bar{\gamma}_{c}, \forall(i, j) \in \mathcal{E} . \bar{\gamma}_{f}, \bar{\gamma}_{v}$ and $\bar{\gamma}_{c}$ are all positive constants. It then follows from (26) that

$$
\left\|\mathbf{e}_{i}(t)\right\| \leq\left[\bar{\gamma}_{f}+\bar{\gamma}_{v}+\bar{\gamma}_{c} \sum_{j=1}^{N} a_{i j}\left(\tilde{\mathbf{x}}_{i}(t)-\tilde{\mathbf{x}}_{j}(t)\right)\right]\left(t-t_{k}^{i}\right) .
$$

Hence, the event triggering function (4) will not be satisfied until the following condition holds:

$$
\begin{gathered}
{\left[\bar{\gamma}_{f}+\bar{\gamma}_{v}+\bar{\gamma}_{c} \sum_{j=1}^{N} a_{i j}\left(\tilde{\mathbf{x}}_{i}(t)-\tilde{\mathbf{x}}_{j}(t)\right)\right]^{2}\left(t-t_{k}^{i}\right)^{2}} \\
=\frac{\sum_{j=1}^{N} a_{i j}\left(\tilde{\mathbf{x}}_{i}-\tilde{\mathbf{x}}_{j}\right)^{T}\left(\tilde{\mathbf{x}}_{i}-\tilde{\mathbf{x}}_{j}\right)+4 \mu e^{-\nu t}}{4 \sum_{j=1}^{N}\left(1+\sigma c_{i j}\right) a_{i j}} .
\end{gathered}
$$

When the state consensus is not yet achieved, we have the right-hand side of (28) is positive, which further implies that the time intervals are strictly positive.

ii) During the interval between two adjacent events of agent $i$, there is at least one of the neighbors of the agent being triggered. Without loss of generality, we assume that one neighbor agent $j$ is triggered after $t_{k}^{i}$ for agent $i$. Then the event triggering instant can be denoted by $t_{l}^{j}$. In other words, $t_{l}^{j}-t_{k}^{i}>0$. Hence, the interval between the $t_{k+1}^{i}-t_{k}^{i}$ is strictly positive.

In summary, we can conclude that the event triggering law can preclude the Zeno behavior and the proof is complete.

Remark 3: Obviously, the global convergence of the algorithm can be guaranteed for any $k_{i j} \in \mathbf{R}_{>0}$. It is not difficult to see that the value of $k_{i j}$ can affect the evolution of adaptive gain $c_{i j}$. It is worth noting that $c_{i j}$ not only affects the control input but also plays a key role in the triggering function. Generally speaking, increasing $k_{i j}$ will achieve a faster convergence rate but meanwhile increase the communication frequency. Therefore, a tradeoff is needed. This observation can be illustrated by numerical simulations in the next section.

\section{Numerical Simulations}

In this section, we illustrate the theoretical results by a numerical simulation. Consider a network system consisting of six agents. Fig. 1 depicts the communication topology among the nodes, which evidently satisfies Assumption 2.

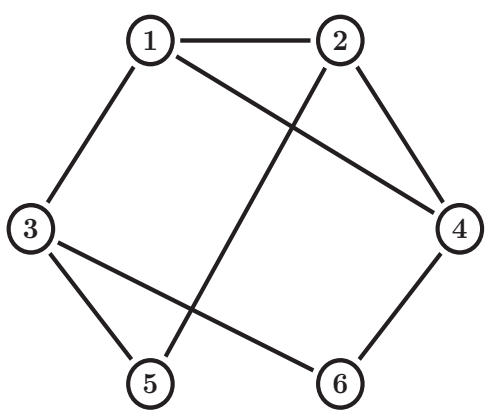

Fig. 1: The communication topology graph.

The local cost functions for each agent are selected as follows: $f_{1}=\sin \left(x_{1}-\frac{\pi}{2}\right), f_{2}=-\cos \left(\ln \left(x_{2}^{2}+2\right)-0.2\right)$, $f_{3}=\left(x_{3}+0.3\right)^{2}+e^{0.3 x_{3}}, f_{4}=0.2 e^{-0.2 x_{4}}+0.4 e^{0.4 x_{4}}$, $f_{5}=\left(x_{5}\right)^{2}\left(\ln \left(x_{5}^{2}+0.5\right)\right)+\left(x_{5}\right)^{2}, f_{6}=\frac{\left(x_{6}\right)^{2}}{\sqrt{\left(x_{6}\right)^{2}+6}}$. The images of these functions are depicted in Figs.2-3. Note that the local cost functions of agents 1,2 are nonconvex. Besides, the local cost functions of agents $3,4,5,6$ are all convex function, and the gradients of $f_{3}$ and $f_{4}$ are locally Lipschitz. It can be verified the strict convexity of the global cost function, which ensures the uniqueness of the optimal solution. The initial states of $\mathbf{x}_{i}(0)$ are chosen randomly within $[-10,10]$. For simplicity, suppose the initial values of $\mathbf{v}_{i}$ and $c_{i j}$ are all equal to zern
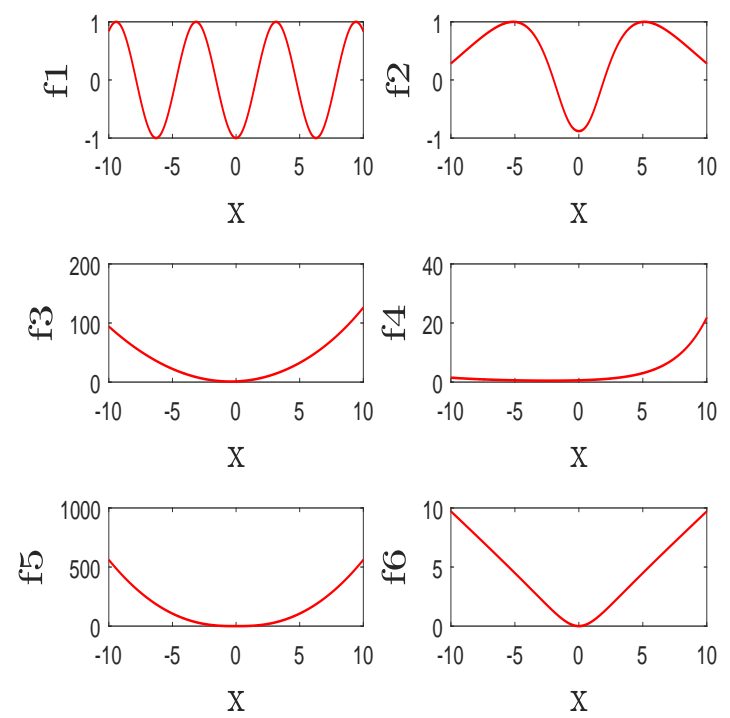

Fig. 2: The images of the local cost functions for different agent $i$. 


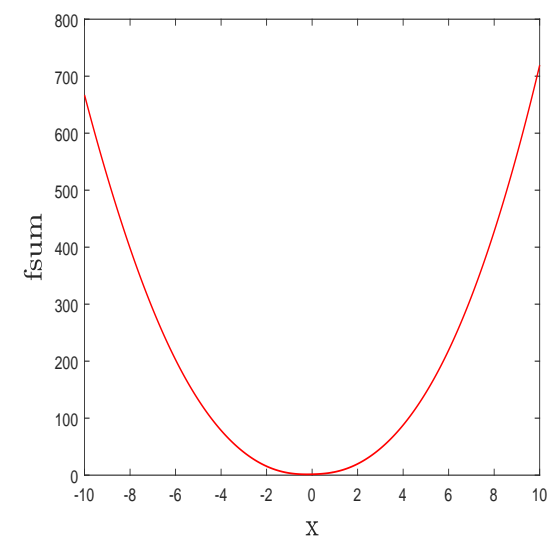

Fig. 3: The images of the goal cost function.
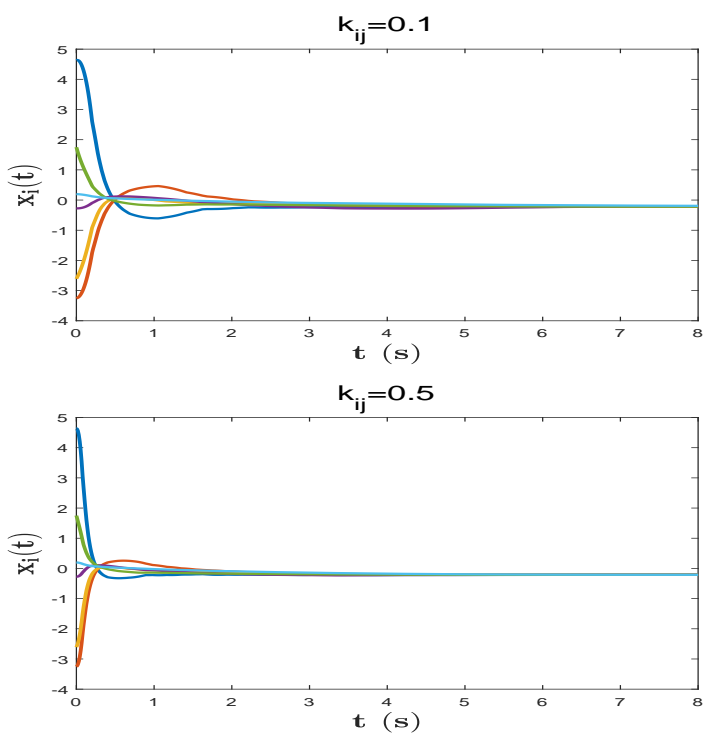

Fig. 4: The trajectories of the agents' states $x_{i}$ for different $k_{i j}$.
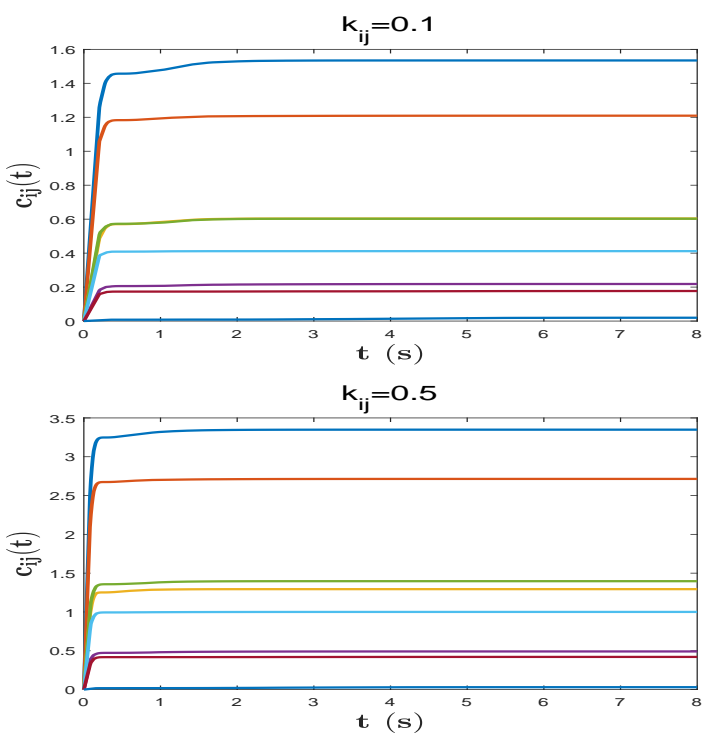

Fig. 5: The trajectories of the adaptive coupling gains $c_{i j}$ for different $k_{i j}$.
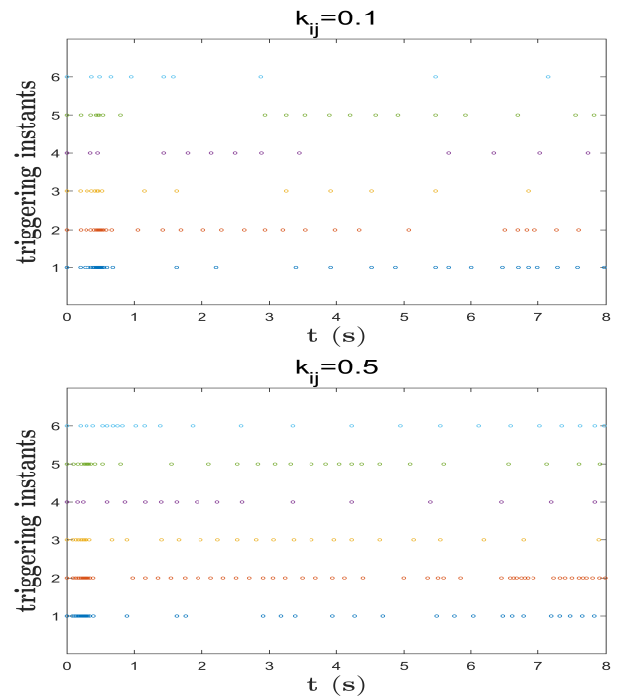

Fig. 6: Triggering instants of each agent, where a circle represents an event.

In order to illustrate how the parameter $k_{i j}$ affects the performance of the event-triggered adaptive algorithm, $k_{i j}=0.1$ and $k_{i j}=0.5$ are considered separately. For both the cases, the trajectories of the agents' states, the time-varying coupling weights $c_{i j}$, and the event time sequence of each agent are depicted in Figs.4-6. Evidently, all the state trajectories asymptotically converge to the global optimal solution and each $c_{i j}$ converges to a finite value. It can be further noticed that smaller $k_{i j}$ implies a slower convergence rate and a reduced communication frequency. This is consistent with the observations in Remark 3.

\section{CONCLUSions}

This paper has addressed the continuous-time DOP with aperiodic communications. One main contribution of this paper comparing the existing literature is that we have presented a novel adaptive consensus-based event-triggering algorithm, which can guarantee the asymptotic convergence to the optimal solution and exclude the Zeno behavior. The proposed adaptive algorithm bases on only the local state information sampled and transmitted at aperiodic time instants determined by a given event triggering function, is fully distributed for the independence of any global information. We may extend the adaptive algorithm to the DOP with time-varying local cost functions in the future.

\section{REFERENCES}

[1] S. H. Low and D. E. Lapsley, "Optimization flow control, I: Basic algorithm and convergence," IEEE/ACM Transactions on Networking, vol. 7, no. 6, pp. 861-874, 1999.

[2] S. Boyd, N. Parikh, E. Chu, B. Peleato, and J. Eckstein, "Distributed optimization and statistical learning via the alternating direction method of multipliers," Foundations and Trends in Machine Learning, vol. 3, no. 1, pp. 1-122, 2010.

[3] A. Cherukuri and J. Cortes, "Initialization-free distributed coordination for economic dispatch under varying loads and generator commitment," Automatica, vol. 74, pp. 183-193, 2016. 
[4] S. S. Ram, V. V. Veeravalli, and A. Nedic, "Distributed and recursive parameter estimation in parametrized linear state-space models," IEEE Transactions on Automatic Control, vol. 55, no. 2, pp. 488-492, 2016.

[5] A. Nedic and A. Ozdaglar, "Distributed subgradient methods for multiagent optimization," IEEE Transactions on Automatic Control, vol. 54, no. 1, pp. 48-61, 2009.

[6] Z. Li and Z. Duan, Cooperative Control of Multi-agent Systems: A Consensus Region Approach. CRC Press, Boca Raton, FL, 2014.

[7] Y. J. Liu, S. Lu, D. Li, and S. Tong, "Adaptive controller design-based ablf for a class of nonlinear time-varying state constraint systems," IEEE Transactions on Systems, Man, and Cybernetics: Systems, vol. 47, no. 7, pp. 1546-1553, 2017.

[8] W. He, G. Chen, Q. L. Han, W. Du, J. Cao, and F. Qian, "Multiagent systems on multilayer networks: Synchronization analysis and network design," IEEE Transactions on Systems, Man, and Cybernetics: Systems, vol. 47, no. 7, pp. 1655-1667, 2017.

[9] G. Wen, P. Wang, T. Huang, W. Yu, and J. Sun, "Robust neuroadaptive containment of multileader multiagent systems with uncertain dynamics," IEEE Transactions on Systems, Man, and Cybernetics: Systems, in press, 2017.

[10] C. L. P. Chen, C.E. Ren, and T. Du, "Fuzzy observed-based adaptive consensus tracking control for second-order multiagent systems with heterogeneous nonlinear dynamics," IEEE Transactions on Fuzzy Systems, vol. 24, no. 4, pp. 906-915, 2016.

[11] Z. Liu, F. Wang, Y. Zhang, and C. L. P. Chen, "Fuzzy adaptive quantized control for a class of stochastic nonlinear uncertain systems," IEEE Transactions on Cybernetics, vol. 46, no. 2, pp. 524-534, 2016.

[12] H. Li, Q. L, X. Liao, and T. Huang, "Accelerated Convergence Algorithm for Distributed Constrained Optimization under Time-Varying General Directed Graphs," IEEE Transactions on Systems Man and Cybernetics Systems, in press, 2017.

[13] H. Li, C. Huang, C. Guo, X. Liao, and T. Huang, "Distributed Consensus Optimization in Multiagent Networks With Time-Varying Directed Topologies and Quantized Communication," IEEE Transactions on Cybernetics, in press, 2017.

[14] P. Bianchi and J. Jakubowicz, "Convergence of a multi-agent projected stochastic gradient algorithm for non-convex optimization," IEEE Transactions on Automatic Control, vol. 58, no. 2, pp. 391-405, 2013.

[15] J. Wang and N. Elia, "A control perspective for centralized and distributed convex optimization," Decision and Control and European Control Conference, pp. 3800-3805, 2011.

[16] J. Lu and C. Y. Tang, "Zero-gradient-sum algorithms for distributed convex optimization: The continuous-time case," IEEE Transactions on Automatic Control, vol. 57, no. 9, pp. 2348-2354, 2012.

[17] B. Gharesifard and J. Cortes, "Distributed continuous-time convex optimization on weight-balanced digraphs," IEEE Transactions on Automatic Control, vol. 59, no. 3, pp. 781-786, 2014.

[18] Y. Zhao, Y. Liu, G. Wen, and G. Chen, "Distributed optimization of linear multi-agent systems: Edge- and node-based adaptive designs," IEEE Transactions on Automatic Control, in press, 2017.

[19] Z. Li, Z. Ding, J. Sun, and Z. Li, "Distributed adaptive convex optimization on directed graphs via continuous-time algorithms," IEEE Transactions on Automatic Control, in press, 2017.

[20] Z. Deng and Y. Hong, "Distributed event-triggered optimization for multi-agent systems with disturbance rejection," IEEE International Conference on Control and Automation, pp. 13-18, 2016.

[21] H. Li, S. Liu, Y. Soh, and L. Xie, "Event-Triggered Communication and Data Rate Constraint for Distributed Optimization of Multiagent Systems," IEEE Transactions on Systems Man and Cybernetics Systems, in press, 2017.

[22] S. S. Kia, J. Cortes, and S. Martinez, "Distributed convex optimization via continuous-time coordination algorithms with discrete-time communication," Automatica, vol. 55, pp. 254-264, 2015.

[23] W. Chen and W. Ren, "Event-triggered zero-gradient-sum distributed consensus optimization over directed networks," Automatica, vol. 65, pp. 90-97, 2016.

[24] D. S. Bernstein, Matrix Mathematics: Theory, Facts, and Formulas (Second Edition). Princeton University Press, 2009.

[25] P. Ioannou and J. Sun, Robust adaptive control. Prentice-Hall, Inc., 1995. 